Original Article (short paper)

\title{
Effects of the aerobic exercise on the learning of a sports motor skill
}

\author{
Giordano Marcio Gatinho Bonuzzi $^{1}$ (D), Érico Jean Moura $^{\text {Alves }}{ }^{1}$ (D), Alaercio Perotti Junior ${ }^{2,3}$ (D) \\ Universidade Estadual do Piauí, Picos, PI, Brasil. \\ Centro Universitário Hermínio Ometto, Araras, SP, Brasil. \\ ${ }^{3}$ Faculdades Integradas Einstein de Limeira, Limeira, SP, Brasil.
}

\begin{abstract}
Aims: This study aimed to investigate the effects of aerobic exercise on the learning of a sports motor skill. Methods: Forty individuals were allocated to the Practice + Exercise Group (PEG) and for the Practice Group (PG). All participants practiced the underhand serve of the Volleyball; all of them were naïve regarding the motor task. The participants (age/ years: PEG: $21.7 \pm 3.06$, PG: $20.25 \pm 1.95$ ) performed a pre-test with five trials, an acquisition phase with fifteen blocks/ 5 trials each, a post-test with five trials, and a twenty-four hours retention test composted by five trials. The PEG was submitted to an aerobic exercise session immediately after the acquisition phase. It was provided by running around the sports court, for 20 minutes, with an intensity of $85 \%$ of the máx heart rate. The PG rest after the acquisition phase. The score and variable errors were the dependent variables. For the motor improvement during the practice, the Anova two way followed by Tukey posthoc test was run, for the retention test was used a T-test, an alpha of 5\% was adopted. Results: All groups increased their score through the practice, with no significant difference between them. In the retention test, the PEG demonstrated better motor performance than PG; it may be related to improvements in the consolidation mechanisms induced by aerobic exercise. Conclusion: The aerobic exercise may be a neuromodulatory strategy to enhance the learning of complex motor skills.
\end{abstract}

Keywords: consolidation, motor learning, aerobic exercise, sports motor skill.

\section{Introduction}

Neuroscientific studies have shown that aerobic exercise can induce transient gradient in the Central Nervous System (CNS) which improves cognitive processing and retention of declarative memories ${ }^{1}$. These findings encouraged researches in order to elucidate the acute effects which the aerobic exercise could have on the procedural memories (motor memories). These preliminary researches have corroborated with the positive findings already mentioned. It has been identified positive effects of the aerobic exercise bout on the motor learning processes in healthy neurologic individuals ${ }^{2,3}$ and neurologic population, as stroke patients ${ }^{4,5}$ and Parkinson disease ${ }^{6,7}$.

In general, the aerobic exercise has been considered with a physical intervention which may upregulate the neurochemical environment resulting: 1 - better motor performance during acquisition phase $\mathrm{s}^{8-10}, 2$ - better cognitive function and information processing during motor practice $^{11}$, and as was used in the item 1 and item 2. Example: 3 - better retention of the improvement caused by the motor practice ${ }^{12-14}$.

These positive effects on the encoding (exposure to the information to be stored, for motor skills learning, the encoding processes are engaged during the practice) and consolidation (when the motor memory trace is transformed in a more robust long term memory; after the encoding processes/ post-practice) of the motor memory are derived from the interaction of several mechanisms, such as the increase of the blood flow and angiogenesis in cortical areas ${ }^{15}$, the rise in the brain volume $e^{16,17}$, the stimulate for production of Brain-Derived Neurotrophic Factor $(\mathrm{BDNF})^{18-21}$, and the increase of neurotransmitters related with vigilance, attention and information processing $^{22}$.

It seems that aerobic exercise can be considered a neuromodulatory strategy that may make the CNS be more sensible to motor memory consolidation mechanisms. However, this finding cannot be applied to all motor skills. Because, all studies which investigated the effects aerobic exercise on the motor learning process used simple motor skills in their methodology, like Serial Reaction Time Task ${ }^{23}$, Grasping Pursuit Task ${ }^{3,12,13,24}$, Sequential Visual Isometric Pinch Task ${ }^{10}$, and Pursuit Rotor Task ${ }^{25}$. These findings cannot be generalized to Activity of Daily Living (ADL's) and sports motor skills; and the principles derived from simple motor skills are not generalized to complex motor skills ${ }^{26}$.

Naturalistic motor actions as sports skills and ADL's are complex, significant, intentional, involve a higher number of degrees of freedom, and including multi-step phases with problem-solving demands across the motor performance ${ }^{27-30}$. In this way, complex motor tasks demand more requirements in the perceptual, cognitive, 
and motor mechanisms. All these characteristics preclude to generalize the principles derived from simple tasks (laboratory perceptual-motor tasks) to complex tasks (sports skills and ADL's) according to the Wulf and Shea's critical review $^{26}$

Thus, the present study aimed to investigate the effect of aerobic exercise on the learning of a sports motor skill. We hypothesized that the participants who perform aerobic exercise immediately after the motor practice would demonstrate better motor performance in the retention test, due to the positive neuromodulatory effects of the aerobic exercise on the consolidation mechanisms.

\section{Materials and Methods}

The Ethics Committee from Hermínio Ometto University Center, Araras SP, Brazil, approved this study; register number 3.259.054. All the participants signed the term of consent.

\section{Participants}

Forty university students participated in this study. As inclusion criteria were adopted: 1 - To be university student, 2- Neuromotor and cognitive conditions for the understanding and execution of the applied tasks during experimental setup, 3 - Medical license to participate in physical activity programs. For exclusion criteria were considered: 1 - Cardiovascular diseases, 2 - Not being able to practice physical activity regarding "Physical Activity Readiness Questionnaire" (PAR-Q), 3 - Not sign the informed consent form, 4 - More than three months of systematic practice of Volleyball (recreational practitioners were included).

To characterize the fitness level of the participants, they responded to the International Physical Activity Questionnaires (IPAQ); besides, we also computed for each participant the number of minutes of high-intensity physical activity/ per week.

\section{Instruments and Tasks}

The task was the underhand serve of the Volleyball, according to Ugrinowitsch and Manoel's study ${ }^{31}$. The task goal was to perform the underhand serve (Figure 1) in the serving zone, getting the ball over the Volleyball net, hitting the ball into the center of the target on the opposing court.

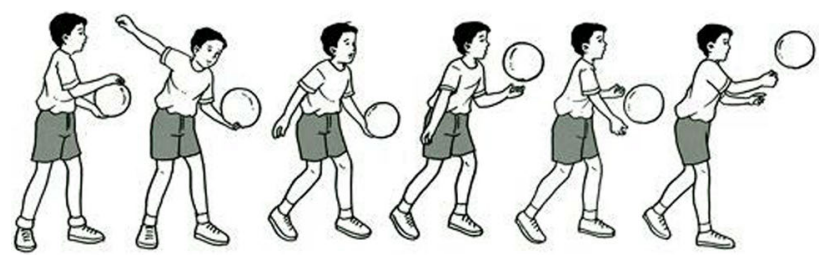

Figure 1 - Underhand serve steps representation.
All the motor practice and the learning tests were performed in an official Volleyball court, in compliance with the international standards of the International Federation of Volleyball. For all experimental setup was used official Volleyball balls (Penalty, 6.0), with calibration between 4.27 to 4.56 pounds. The height of the net was 2.35 meters, without changes during all experimental protocols.

The target on the opposing court was drawn with a $5 \mathrm{~cm}$ wide adhesive tapes on the ground. Thus, the target was composed of 4 squares, which were proportionally interposed, and it had sides of 1 meter, 2 meters, 3 meters, and 4 meters, respectively. A cone 80 centimeters high was allocated for the participant to have a better perception of the center of the target during the serve. A score was recorded when the ball hit the cone or one of the four squares that made up the target, to consider 14 points in case of hit the cone, and $10,8,6$, and 4 points from the most central to the most lateral target, respectively. The participant was positioned at 15 meters from the cone in the middle of the target. The court configuration and target arrangement adopted in the experimental setup can be seen in Figure 2.

\section{Design and procedures}

After the participants sign the Informed Consent Form, they were allocated in the following groups by random processes: Practice + Exercise Group (PEG) and Practice Group (PG) who participated just of motor practice. Both groups performed the same condition of motor practice, however only the PAG after the motor practice participated in an aerobic exercise session. The PG after the motor practice just rested.

The experimental protocol was constituted by two days. On the first day, initially, each participant received instruction on the underhand serve. This instruction was composed of a video demonstrating how the task should be performed. The video showed at a normal speed an experienced volleyball practitioner by a lateral view performing the underhand serve throughout the whole movement. After the video demonstration, the participant performed three trials to familiarization.

Following, the participant performed a pre-test consisted of five trials, an acquisition phase composed of fifteen blocks of five trials, and a post-test formed of five trials. It was provided two minutes of rest among the blocks of the acquisition phase (pre-test, practice blocks, and post-test). On the second day (after twenty-four hours from post-test, approximately) was run the retention test, also composed of five trials. This experimental design was established in a pilot study to determine the ideal amount of practice. For all trials, it was provided feedback (knowledge of result) considering the score achieved on the target. 


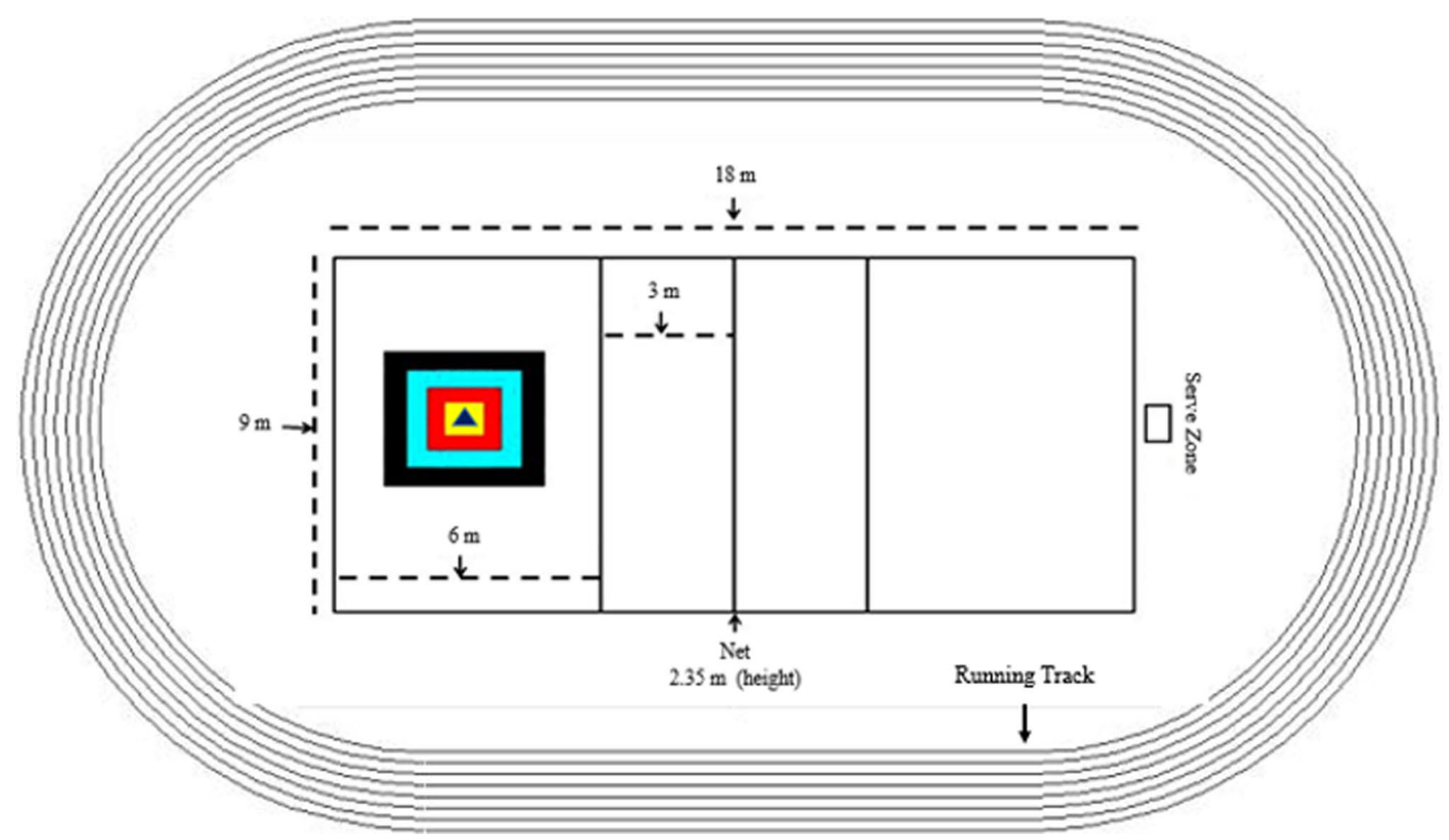

Figure 2 - Schematic representation of the Volleyball court and the running track used in the experiment.

The unique aspect that differentiated the PEG and the PG was that after the motor practice on the first day, the PEG performed an aerobic training session. The aerobic exercise was done in a running track around the sports court where the motor practice occurred, as can be seen in figure 2. The exercise protocol was based on Statton et al.'s study ${ }^{10}$. It consisted of walking for five minutes to warm-up and twenty minutes of running from moderate to intense intensity $(65 \%$ to $85 \%$ of the max heart rate predicted for age). We used this intensity parameter because it is known that moderate protocol can provide positive cognitive effects ${ }^{32}$ and also provide benefits in the long-term memory ${ }^{33}$.

The max heart rate predicted for age was based in Karvonen formula (220-age). Also, it was provided five minutes of walking to cool down after running. During the exercise, it was computed information about the Borg Scale of Perceived Exertion (modified 1 - 10) (BORG 110 ), and the mean and max heart rate (HR) achieved during the exercise prescription was computed by a Polar heart rate monitor, M430 model.

\section{Measures}

Our dependent variables were the score and the variable error; these measures enable the assessment of accuracy and consistency, respectively. The score was determined by the mean points achieved in each trial block. The variable error was calculated by the standard deviation of the points in each trial block.

\section{Statistical analysis}

For the statistical analysis was used the STATISTICA 11.0 (StatSoft Inc., Tulsa, OK, USA) and Microsoft Excel 365. The alpha of 5\% was adopted for the significance level in the inferential analysis. For our dependent variables, we verified the normality and homogeneity using the Shapiro Wilk and Levene tests, respectively; and, the parametric assumptions were identified.

Firstly, we run a T-test to compare the age between the groups. To analyze the minutes involved in high-intensity physical activity/ per week between the groups, we run a Mann-Whitney U test. Also, we performed descriptive analyses for the gender of the participants in each group. As well, we described the perceived exertion (BORG 1-10), the max, minimum, the mean HR, and the mean percentage of the HR achieved during the aerobic exercise provided for PEG.

To assess whether the PEG and PG demonstrated similar motor performance during the acquisition phase, we run an ANOVA two way ( 2 groups (PEG, PG) $x$ 2 times (pre-test, post-test) followed by Tukey posthoc test using the score and variable error. After, we sought to determine the effects of the aerobic exercise on the consolidation processes. For that, we run a T-test comparing the score and variable error on the retention test of the 
PEG and PG. All inferences analyses were performed using the mean (score)/ standard deviation (variable error) of the blocks of five trials from the pre-test, post-test, and retention test.

\section{Results}

We identified that there was no significant difference between PEG and PG for the age $[t(38)=1.79, p>.05]$ and for the number of minutes of high-intensity physical activity/ per week $[t(38)=0.83, p>.05]$. In this way, we suppose that the groups demonstrated homogeneity regarding the age and physical activity level. Also, most participants were male $[\mathrm{PEG}=15, \mathrm{PG}=12]$, and most participants were Very Active regarding the physical activity level $[\mathrm{PEG}=13, \mathrm{PG}=11]$. The demographics information about the PEG and PG's participants can be checked in Table 1.

Concerning the intensity of the exercise that the PEG achieved during the aerobic session, the participants performed aerobic exercise close to $85 \%$ of the máx HR predicted by age. Only five participants considered the aerobic exercise as intense; all others PEG's participants considered the exercise protocol as very intense and very, very intense. The information about the exercise intensity can be verified in Table 2 .

Regarding the score across the acquisition phase, the ANOVA two way detected a significant difference in the Time factor $[F(1)=7.17, p<.05, \mathrm{SMD}=0.32]$, and the post hoc Tukey test showed that the groups improved their

Table 1 - : Participants characterization and demographics

\begin{tabular}{ccc}
\hline & PEG & PG \\
\hline Personal information & & \\
Age (years) & $21.7 \pm 3.06$ & $20.25 \pm 1.95$ \\
Gender & $15 \mathrm{M} / 5 \mathrm{~F}$ & $12 \mathrm{M} / 8 \mathrm{~F}$ \\
Physical Activity Level & & \\
IPAQ - Very Active & 13 & 11 \\
IPAQ - Active & 2 & 3 \\
IPAQ - Irregularly Active A & 2 & 2 \\
IPAQ - Irregularly Active B & 2 & 2 \\
Sedentary & 1 & 2 \\
\# minutes of high-intensity physical & $172.5 \pm 175.5$ & $225 \pm 219.9$ \\
activity/ per week & & \\
\hline
\end{tabular}

Legend: PEG - Practice + Exercise Group; PG - Practice Group; M Male; F - Female; IPAQ - International Physical Activity Questionnaires. Data are presented as mean \pm standard deviation. score between the pre-test and the post-test $[p<.05]$. Also, there was no significant difference between the PEG and PG during the acquisition phase, as demonstrated by the Group factor in the ANOVA two way $[F(1)=2.03, p=$ .16]. In this sense, we can suppose that both groups improved their accuracy in the same magnitude throughout motor practice, as demonstrated in Figure 3 - A.

Concerning variable error, there was no significant difference between PEG and PG across the acquisition phase $[F(1)=1.34, p=.25]$; also, the ANOVA two way did not identify significant difference for the Time factor $[F(1)=1.68, p=.20]$. In this way, our findings demonstrated that the practice did not influence the motor consistency along with the acquisition phase. It can be verified in Figure 3 - B.

About the retention test, the T-test revealed a significant difference in the score $[t(38)=3.73, p<.001]$ between PEG $[M=7.12, S D=1.81]$ and PG $[M=4.9, S D$ $=1.94]$. Also, the T-test demonstrated a significant difference in the variable error $[t(38)=3.53, p<.005]$ between PEG $[M=2.18, S D=0.76]$ and PG $[M=3.17, S D=1.0]$. This finding suggests that aerobic exercise prescription induced improvement of the consolidation mechanisms, which allowed a better motor performance in the retention test, as can been noted in Figure 3 - A and B.

\section{Discussion}

This study aimed to investigate the effects of aerobic exercise on the learning of a sports motor skill. Our findings corroborated our hypothesis. We identified that the participants demonstrated improvement in the accuracy in a similar way along with the motor practice; besides, they did not demonstrate significant differences regarding the consistency of the motor performance. Nevertheless, in the retention test, the PEG showed better accuracy and consistency of the motor performance if compared to PG. This difference probably occurred due to the positive effects of the aerobic exercise on the consolidation processes.

Interestingly, our results as well corroborated with the studies that investigated the effects of the aerobic exercise on motor learning processes using laboratory perceptual-motor tasks. In those was demonstrated a positive impact of the aerobic exercise on motor performance in the retention test using Grasping Pursuit Task ${ }^{3}{ }^{12-14,24}$, Serial Reaction Time Task ${ }^{23}$, and Sequential Visual Isometric Pinch Task ${ }^{10}$.

Table 2 - : Exercise intensity.

\begin{tabular}{cccccc}
\hline Minimum HR* & Mean HR* & Máx HR* & Mean \% máx HR (220 - age) & BORG (1-10) SCORE & BORG (1-10) Classification \\
\hline $171.45 \pm 13.04$ & $177.21 \pm 7.08$ & $181.8 \pm 5,37$ & $84 \% \pm 0,03 \%$ & $8,13 \pm 1,37$ & 6 Very, Very Intense 9 Very Intense 5 Intense
\end{tabular}

Legend: HR, Heart Rate; *, achieved during the aerobic exercise session; BORG (1-10), Borg Scale of Perceived Exertion (modified 1 - 10).

Data are presented as mean \pm standard deviation. 

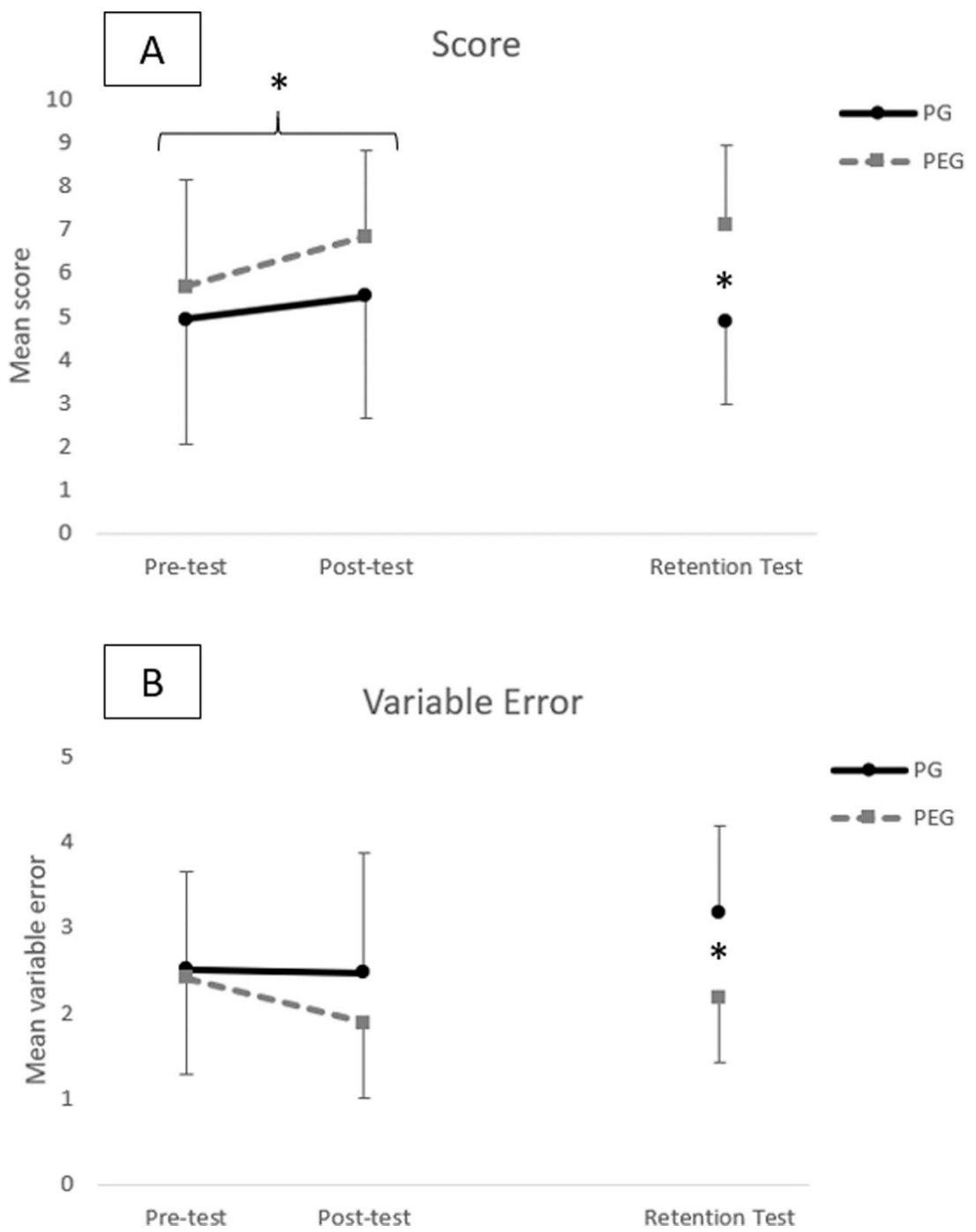

Figure 3 - A - Mean score along with blocks of trials for PEG and PG; B - Mean variable error through blocks of trials for PEG and PG. Legend: PG Practice Group, PEG - Practice + Exercise Group, * - Significant difference. Data are presented as mean \pm standard deviation.

For us, the replicability of the findings from laboratory motor tasks to complex motor tasks is essential; because it allows supposing that the principles observed in simple motor tasks can be generalized to professional intervention contexts, like sports coaching and physiotherapy sessions. In this sense, as far as we have known this study was the first that found positive effects of the acute neuromodulatory effects from the aerobic exercise on learning of a complex motor skill.

There is plausibility on the fact that aerobic exercise can be a neuromodulatory strategy to enhance the learning of complex motor skills, such as sports motor skills or ADL's. It has been supposed that complex motor skills have a higher cognitive demand, given its greater problem-solving requirements ${ }^{26}$. The aerobic exercise may influence this aspect due to the upper-regulation of neurotransmitters (e.g., dopamine) and catecholamines, which may benefit the information processing ${ }^{11,34}$.
Also, complex motor skills engage higher demand in the motor system, given the involvement of a higher number of degrees of freedom ${ }^{26,30}$. This condition induces a more significant challenge in the creation of synergies for interlimb coordination ${ }^{35}$. We suppose that aerobic exercise can facilitate this mechanism by providing excitability in the motor cortex ${ }^{36}$. This condition probably may impact the movement pattern positively during the motor learning processes.

Besides, sports motor skills and ADLs demand more perceptual requirements if compared to simple motor tasks, in particular for discrimination and information selection $^{30}$. Again, the aerobic exercise can facilitate this mechanism, as demonstrated by Perini et al. study ${ }^{37}$, which revealed that a moderate aerobic exercise produced better perceptual discrimination.

Especially in our study, we presume that the aerobic exercise influenced mainly the memory creation pro- 
cesses, more specifically the consolidation process because 1 - we identified that both groups were equal at the end of the motor practice, demonstrating a similar condition of encoding mechanisms. 2 - we provided the exercise immediately after the motor practice, what can be influenced only the consolidation mechanisms that occur in wakefulness moment post-practice ${ }^{38}$ and during the night sleep ${ }^{39,40}$.

Another important aspect regarding the comparison of our findings with the previous studies that found positive effects of the aerobic exercise on the consolidation processes of motor memories is the intensity of the exercise. The majority of prior studies used High-Intensity Interval Training (HIIT) protocols $s^{3,12-14,24,41,42}$. In contrast, in our protocol, we used a continuous moderate aerobic exercise.

In this sense, maybe the neuromodulatory effects from the aerobic exercise are also achieved in moderate protocols, not limited only to HIIT protocols. Including, Baird et al. ${ }^{43}$ did not found a significant difference in the effects on the motor learning between intense and moderate energy-matched exercise intensity protocols during the learning of a laboratory serial target task. In this sense, in practical intervention contexts, maybe both protocols (HIIT and continuous moderate/ intense) can be used to enhance motor learning processes. New investigations in this area are promising and may assist in the proper structuring of motor interventions that are combined with physical fitness sessions.

We consider as a limitation of our study the fact that we did not have outcomes related to the movement pattern. All our inferences were based on the result of the movement, in other words, the score. Until now, there is no evidence about the effects of the aerobic exercise prescription on the movement pattern during the learning of a naturalistic motor skill. For us, it is an interesting issue for the subsequent studies in this area.

Another aspect that we consider a limitation of our study was related to the retention test. For us, a long-term retention test could add more information about the consolidation mechanisms, once that the 24 hours retention test reveals the consolidation mechanisms status and longterm retention (like a 1-month retention test) test could offer information about the forgetfulness process. For example, Roig et al. ${ }^{13}$ demonstrated no difference between the exercise group and control group in the motor performance of a visuomotor accuracy-tracking task in the 24 hours retention test. However, they identified the superiority of the exercise group in a long-term retention test (1 week). Probably the information about short-term and long-term retention tests are complementary, and it is suggested to have them both in the ensuing investigations.

Besides that, for a better comprehension of the consolidation processes is recommended to have savings measures. 'Savings' refers to a more rapid rate of relearn- ing compared with the rate of original learning ${ }^{44}$. Retention tests are commonly done in a few trials to avoid warmup or fatigue effects on what was possibly retained $^{45}$. However, retention tests with a few trials only assess the absolute retention (how much it has been retained from the motor performance improved in the acquisition phase), and it neglects the relative retention (how much practice could be saved to achieve a criterion performance) ${ }^{46}$. In this sense, whether during the retention test the performance criteria is reacquired more quickly than it was done in the acquisition phase, it can be assumed that something was retained from the previous practice, supporting this faster relearning ${ }^{46,47}$.

In our study, we have just a few trials in the retention test; then, we did not assess the savings to evaluate the relative retention. Maybe it can be valuable in inferences about the effects of aerobic exercise on the consolidation of motor memories. For example, Mang et al. ${ }^{42}$ did not identify a significant difference between the Exercise + Practice Group and the Practice Group during the learning of a Serial Reaction Time Task. However, they verified that the Exercise + Practice Group demonstrated a faster saving, which suggests a better consolidation if compared to the Practice Group.

Therefore, we suggest more studies investigating the effects of the aerobic exercise bout in the learning of naturalistic motor skills to verify whether the findings derived from laboratory perceptual-motor skills are generalized to complex motor skills. It is advised that the next researches in this subject incorporates movement patterns measures (i.e., kinematic and kinetic analyses), long-term retention tests, and savings analyses.

\section{Conclusion}

The aerobic exercise can enhance the learning of a sports motor skill. This improvement may be attributed to the neuromodulatory effects of the aerobic exercise on the upper regulation of consolidation mechanisms from motor memories.

\section{References}

1. Hillman $\mathrm{CH}$, Erickson KI, Kramer AF. Be smart, exercise your heart: exercise effects on brain and cognition. Nat Rev Neurosci. 2008;9(1):58-65. doi: 10.1038/nrn2298

2. Mang CS, Brown KE, Neva JL, Snow NJ, Campbell KL, Boyd LA. Promoting motor cortical plasticity with acute aerobic exercise: a role for cerebellar circuits. Neural Plast. 2016;2016:6797928. doi: 10.1155/2016/6797928

3. Roig M, Thomas R, Mang CS, Snow NJ, Ostadan F, Boyd LA, et al. Time-dependent effects of cardiovascular exercise on memory. Exerc Sport Sci Rev. 2016;44(2):81-88. doi: 10.1249/JES.0000000000000078

4. Nepveu J, Thiel A, Tang A, Fung J, Lundbye -Jensen J, Boyd LA, et al. A single bout of high-intensity interval trai- 
ning improves motor skill retention in individuals with Stroke. Neurorehabil Neural Repair. 2017;31(8):726-735. doi: $10.1177 / 1545968317718269$

5. Quaney BM, Boyd LA, McDowd JM, Zahner LH, He J, Mayo MS, et al. Aerobic exercise improves cognition and motor function Poststroke. Neurorehabil Neural Repair. 2009;23(9):879-885. doi: 10.1177/1545968309338193

6. Duchesne C, Lungu O, Nadeau A, Robillard ME, Boré A, Bobeuf F, et al. Enhancing both motor and cognitive functioning in Parkinson's disease: Aerobic exercise as a rehabilitative intervention. Brain Cogn. 2015;99:68-77. doi: 10.1016/j.bandc.2015.07.005

7. Petzinger GM, Fisher BE, McEwen S, Beeler JA, Walsh JP, Jakowec MW. Exercise-enhanced neuroplasticity targeting motor and cognitive circuitry in Parkinson's disease. Lancet Neurol. 2013;12(7):716-726. doi: 10.1016/S1474-4422(13) 70123-6

8. Kashihara K, Nakahara Y. Short-term effect of physical exercise at lactate threshold on choice reaction time. Percept Mot Skills. 2005;100(2):275-291. doi: 10.2466/ pms.100.2.275-291

9. Snow NJ, Mang CS, Roig M, McDonnell MN, Campbell KL, Boyd LA. The effect of an acute bout of moderateintensity aerobic exercise on motor learning of a continuous tracking task. PLoS One. 2016;11(2):1-16. doi: 10.1371/ journal.pone.0150039

10. Statton MA, Encarnacion M, Celnik P, Bastian AJ. A single bout of moderate aerobic exercise improves motor skill acquisition. PLoS One. 2015;10(10):e0141393. doi: 10.1371/journal.pone. 0141393

11. McDonnell MN, Smith AE, Mackintosh SF. Aerobic exercise to improve cognitive function in adults with neurological disorders: a systematic review. Arch Phys Med Rehabil. 2011;92(7):1044-1052. doi: 10.1016/j.apmr.2011.01.021

12. Mang CS, Snow NJ, Campbell KL, Ross CJD, Boyd LA. A single bout of high-intensity aerobic exercise facilitates response to paired associative stimulation and promotes sequence-specific implicit motor learning. J Appl Physiol. 2014;117(11):1325-1336. doi: 10.1152/japplphysiol.00498.2014

13. Roig M, Skriver K, Lundbye-Jensen J, Kiens B, Nielsen JB. A single bout of exercise improves motor memory. PLoS One. 2012;7(9):e44594. doi:10.1371/journal.pone.0044594

14. Skriver K, Roig M, Lundbye-Jensen J, Pingel J, Helge JW, Kiens B, et al. Acute exercise improves motor memory: Exploring potential biomarkers. Neurobiol Learn Mem. 2014;116:46-58. doi: 10.1016/j.nlm.2014.08.004

15. Taubert M, Villringer A, Lehmann N. Endurance exercise as an "endogenous" neuro-enhancement strategy to facilitate motor learning. Front Hum Neurosci. 2015;9. doi: 10.3389/ fnhum.2015.00692

16. Kleim JA, Cooper NR, VandenBerg PM. Exercise induces angiogenesis but does not alter movement representations within rat motor cortex. Brain Res. 2002;934(1):1-6. doi: 10.1016/S0006-8993(02)02239-4

17. Smith PJ, Blumenthal JA, Hoffman BM, Cooper H, Strauman TA, Welsh-Bohmer K, et al. Aerobic exercise and neurocognitive performance: a meta-analytic review of randomized controlled trials. Psychosom Med. 2010;72 (3):239-252. doi: 10.1097/PSY.0b013e3181d14633
18. Cotman C. Exercise: a behavioral intervention to enhance brain health and plasticity. Trends Neurosci. 2002;25 (6):295-301. doi: 10.1016/S0166-2236(02)02143-4

19. Knaepen K, Goekint M, Heyman EM, Meeusen R. Neuroplasticity?? Exercise-induced response of peripheral brainderived neurotrophic factor. Sport Med. 2010;40(9):765801. doi: 10.2165/11534530-000000000-00000

20. Marlatt MW, Lucassen PJ, van Praag H. Comparison of neurogenic effects of fluoxetine, duloxetine and running in mice. Brain Res. 2010;1341:93-99. doi: 10.1016/j. brainres.2010.03.086

21. Rasmussen P, Brassard P, Adser H, Pedersen MV, Leick L, Hart E, et al. Evidence for a release of brain-derived neurotrophic factor from the brain during exercise. Exp Physiol. 2009;94(10):1062-1069. doi: 10.1113/expphysiol.2009.048512

22. McMorris T, Collard K, Corbett J, Dicks M, Swain JP. A test of the catecholamines hypothesis for an acute exercise-cognition interaction. Pharmacol Biochem Behav. 2008;89(1):106-115. doi: 10.1016/j.pbb.2007.11.007

23. Ostadan F, Centeno C, Daloze J-F, Frenn M, Lundbye-Jensen J, Roig M. Changes in corticospinal excitability during consolidation predict acute exercise-induced off-line gains in procedural memory. Neurobiol Learn Mem. 2016;136:196-203. doi: 10.1016/j.nlm.2016.10.009

24. Stavrinos EL, Coxon JP. High-intensity interval exercise promotes motor cortex disinhibition and early motor skill consolidation. J Cogn Neurosci. 2017;29(4):593-604. doi: 10.1162/jocn_a_01078

25. Tomporowski PD, Pendleton DM. Effects of the timing of acute exercise and movement complexity on young adults' psychomotor learning. J Sport Exerc Psychol. 2018;40 (5):240-248. doi: 10.1123/jsep.2017-0289

26. Wulf G, Shea $\mathrm{CH}$. Principles derived from the study of simple skills do not generalize to complex skill learning. Psychon Bull Rev. 2002;9(2):185-211. doi: 10.3758/ BF03196276

27. Schaefer SY, Patterson CB, Lang CE. Transfer of training between distinct motor tasks after stroke: implications for task-specific approaches to upper-extremity neurorehabilitation. Neurorehabil Neural Repair. 2013;27(7):602-612. doi: $10.1177 / 1545968313481279$

28. Schaefer SY, Lang CE. Using dual tasks to test immediate transfer of training between naturalistic movements: a proof-of-principle study. J Mot Behav. 2012;44(5):313-327. doi: 10.1080/00222895.2012.708367

29. Hartmann K, Goldenberg G, Daumuller M, Hermsdorfer J. It takes the whole brain to make a cup of coffee: The neuropsychology of naturalistic actions involving technical devices. Neuropsychologia. 2005;43(4):625-637. doi: 10.1016/j.neuropsychologia.2004.07.015

30. Ingram JN, Wolpert DM. Naturalistic approaches to sensorimotor control. Prog Brain Res. 2011;191:3-29. doi: 10.1016/B978-0-444-53752-2.00016-3

31. Ugrinowitsch H, Manoel EDJ. Interferência contextual: Variação de programa e parâmetro na aquisição da habilidade motora saque do voleibol. Rev Paul Educ física. 1999;13(2):197-216. 
32. McMorris T, Tomporowski PD, Audiffren M. Exercise and Cognitive Function. Chichester, UK: John Wiley \& Sons, Ltd; 2009. doi: 10.1002/9780470740668

33. Labban JD, Etnier JL. Effects of acute exercise on longterm memory. Res Q Exerc Sport. 2011;82(4):712-21. doi: 10.1080/02701367.2011.10599808

34. McMorris T. Exercise and Cognitive Function: A Neuroendocrinological Explanation. In: McMorris T, Tomporowski PD, Audiffren M, eds. Exercise and Cognitive Function. Chichester, UK: John Wiley \& Sons, Ltd; 2009:41-68. doi: 10.1002/9780470740668.ch2

35. Latash ML. Synergy. New York, NY: Oxford University Press; 2008. doi: 10.1093/acprof:oso/ 9780195333169.001.0001

36. Singh AM, Staines WR. The effects of acute aerobic exercise on the primary motor cortex. J Mot Behav. 2015;47 (4):328-339. doi: 10.1080/00222895.2014.983450

37. Perini R, Bortoletto M, Capogrosso M, Fertonani A, Miniussi C. Acute effects of aerobic exercise promote learning. Sci Rep. 2016;6(1):25440. doi: 10.1038/srep25440

38. Cohen DA, Pascual-Leone A, Press DZ, Robertson EM. Off-line learning of motor skill memory: A double dissociation of goal and movement. Proc Natl Acad Sci. 2005;102 (50):18237-18241. doi: 10.1073/pnas.0506072102

39. King BR, Hoedlmoser K, Hirschauer F, Dolfen N, Albouy G. Sleeping on the motor engram: The multifaceted nature of sleep-related motor memory consolidation. Neurosci Biobehav Rev. 2017;80(October 2016):1-22. doi: 10.1016/j. neubiorev.2017.04.026

40. Christova M, Aftenberger H, Nardone R, Gallasch E. Adult gross motor learning and sleep: is there a mutual benefit? Neural Plast. 2018;2018:1-12. doi: 10.1155/2018/3076986

41. Thomas R, Johnsen LK, Geertsen SS, Christiansen L, Ritz $\mathrm{C}$, Roig $\mathrm{M}$, et al. Acute exercise and motor memory consolidation: the role of exercise intensity. PLoS One. 2016;11 (7):e0159589. doi: 10.1371/journal.pone.0159589
42. Mang CS, Snow NJ, Wadden KP, Campbell KL, Boyd LA. High-intensity aerobic exercise enhances motor memory retrieval. Med Sci Sport Exerc. 2016;48(12):2477-2486. doi: 10.1249/MSS.0000000000001040

43. Baird JF, Gaughan ME, Saffer HM, Sarzynski MA, Herter TM, Fritz SL, et al. The effect of energy-matched exercise intensity on brain-derived neurotrophic factor and motor learning. Neurobiol Learn Mem. 2018;156(April):33-44. doi: $10.1016 /$ j.nlm.2018.10.008

44. Krakauer JW, Shadmehr R. Consolidation of motor memory. Trends Neurosci. 2006;29(1):58-64. doi: 10.1016/j. tins.2005.10.003

45. Magill RA, Anderson DI. Motor learning and control: concepts and applications. Vol 11th. New York, NY: McGrawHill Education; 2017.

46. Christina RW. Concerns and issues in studying and assessing motor learning. Meas Phys Educ Exerc Sci. 1997;1 (1):19-38. doi: 10.1207/s15327841mpee0101_2

47. Schmidt R, Lee TD. Motor control and learning: from principles to application. 5th ed. Champaign: Human Kinetics; 2014.

\section{Corresponding author}

Giordano Marcio Gatinho Bonuzzi. Universidade Estadual do Piaui, Picos, PI, Brasil.

E-mail: giordanomgb@gmail.com

Manuscript received on February 2, 2020

Manuscript accepted on March 26, 2020

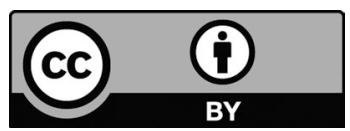

Motriz. The Journal of Physical Education. UNESP. Rio Claro, SP, Brazil - eISSN: 1980-6574 - under a license Creative Commons - Version 4.0 